\title{
Towards standardising irrigation DSS inputs data formats through adaptation of the WDTF/WaterML
}

\author{
$\underline{\text { N. J. Car }}^{\mathrm{a} b}$, G. A. Moore ${ }^{\mathrm{b}}$

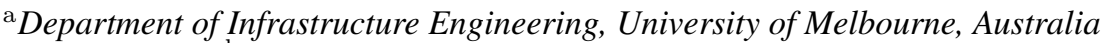 \\ ${ }^{\mathrm{b}}$ CSIRO Land \& Water, Griffith NSW, Australia \\ Email: nicholas.car@csiro.au
}

\begin{abstract}
Irrigators use many data sources such as automatic weather stations (AWS), flow metres, soil moisture probes etc. to inform them when they try to make objective decisions at the farm level. Crop models and irrigation Decision Support Systems (DSS) that they could use to help them in this are hindered by their ability to use the data from all of these sources which diminishes their relevance to irrigators and reduces their uptake. Factors causing this hindrance are:
\end{abstract}

- Physical connectivity access to data;

- Data ownership restrictions;

- Incompatibility of the data formats;

- Unsatisfactory or non-existent quality assurance and metadata for reliable use.

The Internet and wide radio and cellular network coverage across Australia's irrigation districts has greatly improved the physical connectivity aspect of data access while ownership restriction on access have no technical solution. This paper addresses the remaining issues through discussing on-farm irrigation-specific adaptations or extensions, particularly relating to evapotranspiration, of the Bureau of Meteorology's (BoM) currently implemented Water Data Transfer Format (WDTF) described by Walker et al. (2009), the international WaterML project described by Zaslavsky et al. (2007) and their joint future WaterML 2.0 standard as described by Taylor (2009). These projects have evolved rapidly since the authors' previous work on irrigation data standardisation (see Car et al. (2009)) and are now seeing large-scale implementation in Australia and thus immediate adaptation and adoption is now possible. A trial adoption of the extended standard is described in this paper whereby by data from two different AWS networks are able to be used by a previous DSS built by one of the authors and others (see Hornbuckle et al. (2009) for the DSS details).

In addition to the technical implementation of data formats at the network scale, implementation by irrigation-related hardware vendors is discussed. This is crucial to the acceptance of standards for use at the farm scale. Irrigation sensors and other hardware must be natively standards compliant - i.e. not requiring further software layers to be so - as irrigation model and DSS users at this scale will not have the ability to undertake further development.

Specifically, on-farm DSS, irrigation model and WDTF/WaterML background is given in Section 1, conceptual work relating to on-farm irrigation model requirements in Section 2, test implementations of format extensions in Section 3 and case studies of hardware vendor adoption in Section 4.

This proposed exercise in standardisation is part of a larger project by the authors looking to improve the utilisation of on-farm DSS through both technical and non-technical methods. Increased DSS use for onfarm irrigation management decisions not only assists irrigators to use more science in their decisions but also enables decision data to be captured which can then be used post-event by researchers for analysis.

Keywords: Decision Support Systems, irrigation, Water Data Transfer Format, WaterML, standards 


\section{INTRODUCTION}

One of the reasons irrigators don't use irrigation DSS, despite their proved yield benefits Inman-Bamber et al. (2005), is that the systems do not work with data sources with which they are already familiar. For example, if the irrigator already uses an automatic weather station (AWS) and is happy with it's data, they may want to use it, rather than a more remote or modelled station, with a DSS. This could easily be the case with irrigators who live in South Australia and use the SA NRM Board AWSs ${ }^{1}$ but wish now to use the crop model APSIM APSRU (2011). APSIM cannot currently accept data from these AWSs as inputs and instead relies on data interpolated weather data from the SILO project ${ }^{2}$. Not only may the irrigator believe that his local data source reflects his particular weather more accurately than others but the it may also use different calculations for derived data to the remote source. In thecase of the SA NRM Board's automatic AWSs (AWSs) report $A S C E_{\text {short }}$ and $A S C E_{\text {tall }}$ Walter et al. (2000) calculations for evapotranspiration where as SILO mainly use FAO56 Allen et al. (1998) calculations. A similar situation may occur with DSS that uses flow meters of certain brands where an irrigator already has a different brand installed, perhaps at great cost.

The only two options open to DSS designers are to write drivers for every conceivable data source or to fix their data input requirements to a standard to which data providers, both large-scale data providers and one-off equipment manufacturers, adhere. Since the first option is extremely costly and will never reach to include all manufacturers' equipment, the second option is the only one practical. Large-scale data providers, like the BoM are currently standardising data for internal purposes and in initial discussions with industry, key players in the Australian irrigation sector, mentioned in the Acknowledgements Section, are interested in standardisation.

Unlike in 2009, when the authors first wrote about this issue Car et al. (2009), there is now a single, widely used standard for many water measurements that can readily be extended to incorporate irrigation data source-relevant standard specifications. Water data, not specifically relating to irrigation, is collected by a Australian government agencies and must be reported to the BoM in accordance with the Water Act 2007 (C'wealth Gov't of Aust (2007)). To handle this data, CSIRO and the BoM have developed the Water Data Transfer Format (WDTF) which is a set of data and metadata specifications for water observations that must be followed by all water-reporting agencies. This format is mandated for use on a very large scale and surpasses any previous water data-related formats in both it's implementation extent and comprehensiveness and is therefore an ideal candidate base specification for use by the diverse irrigation sector. This standard is being developed with organisations working on hydrological standards in the USA and elsewhere, Taylor (2009) to be compliant with the Open Geospatial Consortium. This ensures adherence to these standards will be of future international relevance.

The WDTF documentation ${ }^{3}$ specifies how water observations and some meteorological data need to be reported so that the readings are understood by the BoM. Sufficient metadata is required to allow the BoM to understand the readings unambiguously without them having knowledge of the measurer's techniques. Examples of this metadata are acceptable measurement units and their descriptors to be used in plain text mark-up ( $m$ for metre, $M L / m o_{c}$ for Megalitres per calendar month etc.), interpolation methods to be used between data points (InstVal for instantaneous values, PrecTot for the total in the preceding interval) as well as which coordinate systems and height data are to be used to express location. WaterML operates in a similar fashion but the standard also provides Internet-based data delivery methods with which all conforming datasets, as long as it is registered with WaterML's developers at the Consortium of Universities for the Advancement of Hydrologic Science (CUAHSI) ${ }^{4}$.

\section{ON-FARM IRRIGATION MODEL AND DSS REQUIREMENTS OF STANDARDS}

Listing all input requirements of all irrigation models at the farm scale is not possible however categories of common inputs can easily be recognised and their relation to the WDTF/WaterML specified. At the most general level, many on-farm irrigation models and DSS need information relating to:

\footnotetext{
${ }^{1}$ See http://www.aws-samdbnrm.sa.gov.au for a station list and area map.

${ }^{2}$ http://www.longpaddock.qld.gov.au/silo/

${ }^{3}$ The latest version of the documentation can be downloaded from ftp://ftp.bom.gov.au/anon/home/water/WDTF/

${ }^{4} \mathrm{http} / / /$ his.cuahsi.org/wofws.html
} 

- crops
- soils
- weather
- water

The WDTF and WaterML do not handle crop or soil data. They either do or will handle most of the water data requirements and some of the weather data requirements that irrigation models and DSS have. Crop data needs to be handled by the modellers and DSS designers. Usually, as is the case with APSIM, this data is either supplied with the model or is the subject of model research and therefore not expected to be reported from sensors. Soils data is often a one-time model input per model location and remains static. Soils data is currently the subject of a separate Australia-wide research project with and delivery through the CSIRO's Australian Soil Resource Information System (http://www.asris.csiro.au). Data from this system can be used by APSIM-based models and other models requiring soil data could used an adapted form of it too. While it is desirable that this data be presented in a fashion similar to data in the WDTF/WaterML, it is outside the scope of this paper to examine the issues involved.

Water and weather data make up the majority of farm-level irrigation model and DSS timeseries data and is commonly able to be sourced locally with local sensors such as weather stations and flow meters.

\subsection{Weather data}

Currently the BoM mandates incoming weather data to be in the WDTF standard and, although it provides copious weather data for individual use, it does not do so in the WDTF format. The first requirement then is that it does so in a manner similar to the WaterOneFlow routines used by CUAHSI in delivering their WaterML data. This may be planned for the future WaterML 2.0. Even if this were to happen, there are several deficiencies with the format - from the point of view of farm level irrigation - that mean it cannot solely be used as inputs to irrigation models or DSS at that level. Most apparent is that the WDTF does not adequately handle evapotranspiration (ET) which is critical for most soil/plant/water-based irrigation models and DSS. The WDTF specifies a single type of ET reading while, in practice, ET values are calculated in a number of ways which include evaporation pan measured values, the FAO56 methodology or variants such as Penman-Montieth and Penman-Meyer or the more recent ASCE methodologies. Since these readings vary widely, additional metadata fields must be added to the WDTF to describe which methodology is implemented. Given the evolving nature of ET equations and continued arrival of new ET techniques, such as the recent Matt-Shuttleworth methodology, Shuttleworth and Wallace (2009), it is more appropriate to add further fields of metadata to the constituent data underlying ET calculations and thus allow data clients to determine what equations can be used, based on what constituent data is present. Table 1 indicates possible metadata fields that could be added to the current WDTF 1.1 standard. If this is done, ET could then be calculated using several methodologies from currently public BoM data as well as data frorm many other publicly available AWS networks such as those in Section 3.

In addition to collecting weather data from national or large AWS networks, irrigators may have access to individual AWSs. For this reason and as its the case with water flow sensors, AWSs should also output data directly into a WDTF/WaterML-like format. This is discussed in Section 3.

\subsection{Water data}

Currently the BoM requires incoming water data from 3rd parties be in the WDTF format but does not provide water data back to individual users in this. This may also change as the WDTF format moves to WaterML 2.0. Since the WDTF/WaterML standards are very comprehensive, it is possible that all farmlevel water flow and water storage volume measurements are catered for within the standard however the majority of water data that individual irrigators can expect to want to input into their models and DSS will be collected from local sensors. These sensors should output their data according to standards already in place. The Open GIS Sensor Observation Service ${ }^{5}$ is best chosen for this due to WaterML 2.0's conformance with it through the OGC standards.

${ }^{5}$ http://www.opengeospatial.org/standards/sos 
Table 1. Possible ET-related metadata additions to the WDTF

\begin{tabular}{|c|c|c|}
\hline Metadata field & Description & Examples \\
\hline ET equation name & $\begin{array}{l}\text { Names the ET equation used for single ET } \\
\text { values }\end{array}$ & $\begin{array}{l}\text { "fao56", "penman-meyer", } \\
\text { "asce-short"... }\end{array}$ \\
\hline Wind height & $\begin{array}{l}\text { Gives the height of windspeed measure- } \\
\text { ments. This would allow measurements at } \\
\text { the same location but multiple height to be } \\
\text { given as well as informing the user as to } \\
\text { likely crop/wind interactions }\end{array}$ & numerical values, in metres \\
\hline Coordinates & $\begin{array}{l}\text { The coordinates of the measuring point if } \\
\text { it is a named point (i.e not originally ac- } \\
\text { cessed via coordinates). This allows for } \\
\text { the calculation of extraterrestrial radiation } \\
\left(R_{a}\right) \text { via the sunset hour angle }\left(\omega_{s}\right)\end{array}$ & $\begin{array}{l}\text { latitude \& longitude in decimal } \\
\text { degrees or other accepted units }\end{array}$ \\
\hline Site elevation & $\begin{array}{l}\text { The height of the named point allowing for } \\
\text { atmospheric pressure calculation }\end{array}$ & altitude in metres \\
\hline Assumed Constants & $\begin{array}{l}\text { Key/value pairs of assumed constants that } \\
\text { may be used, such as the Latent Heat of } \\
\text { Vaporization }(\lambda)\end{array}$ & "lambda" \& $2.45 M J \mathrm{~kg}^{-1}$ \\
\hline
\end{tabular}

\subsection{Connection requirements}

In addition to the data requirements in the preceding subsections, irrigation models and DSS need connection or transmission additions to the WDTF standard. This is because, unlike single entities like the BoM with single data collection points, individual irrigators would need a standard to detail how to establish both delivery and reception points for data in order that communication local sensor $\rightarrow$ model/DSS and remote sensor $\rightarrow$ model/DSS may both be designed for by sensor and model/DSS makers. Currently organisations required to transmit data to the BoM do so using connection details supplied outside the WDTF standard. Such details need to be communicated in a regular way within the standard. Web services, as implemented by WaterOneFlow routines and WaterML 2.0 may not solve this issue if individual sensors are not able to be programmed with Web Service routines. Direct HTML RERSTful $^{6}$ routines may be more applicable as any sensor that can currently connect to the Internet for perhaps FTP data delivery, as many now can, could implement RESTful data delivery with minor modifications. A live, RESTful implementation of mocked-up data for a single AWS is given at http://www.awssamdbnrm.sa.gov.au/wdtf/single.

\section{TEST IMPLEMENTATIONS}

\subsection{Weather data from AWS networks}

Two different AWS networks using different underlying AWS hardware and network configuration were adapted to provide weather data in WDTF-like formats as test input for irrigation models and DSS. The adaptation from WDTF to a future WaterML 2.0 would be straightforward.

Network 1, CSIRO Land \& Water: this network consists of 11 AWSs in New South Wales reporting data hourly to a central location. Before standardisation, data was available via standard web pages at http://weather.irrigateway.net and via Comma Separated Value file downloads only. After minimal programming, the data is now also available in two more formats: 1. online modified WDTF data which can be retrieved via direct webpage access (in XML format) and 2. Web Service-request modified WDTF. Figure 3.1 shows a table of values for a daily reading of values from the Griffith AWS for the $21^{\text {st }}$ of July, 2011 and fragments of WDTF-like marked-up data for the same day as accessed through the first of the new formats. The full daily data for this AWS in WDTF-like syntax is given online at

\footnotetext{
${ }^{6}$ See Wikipedia for a general description or IBM's description for more details.
} 
http://111.118.171.88/wdtf/. Contained there are also versions of the data in pure WDTF and modified WDTF formats: with and without the additional metadata components.

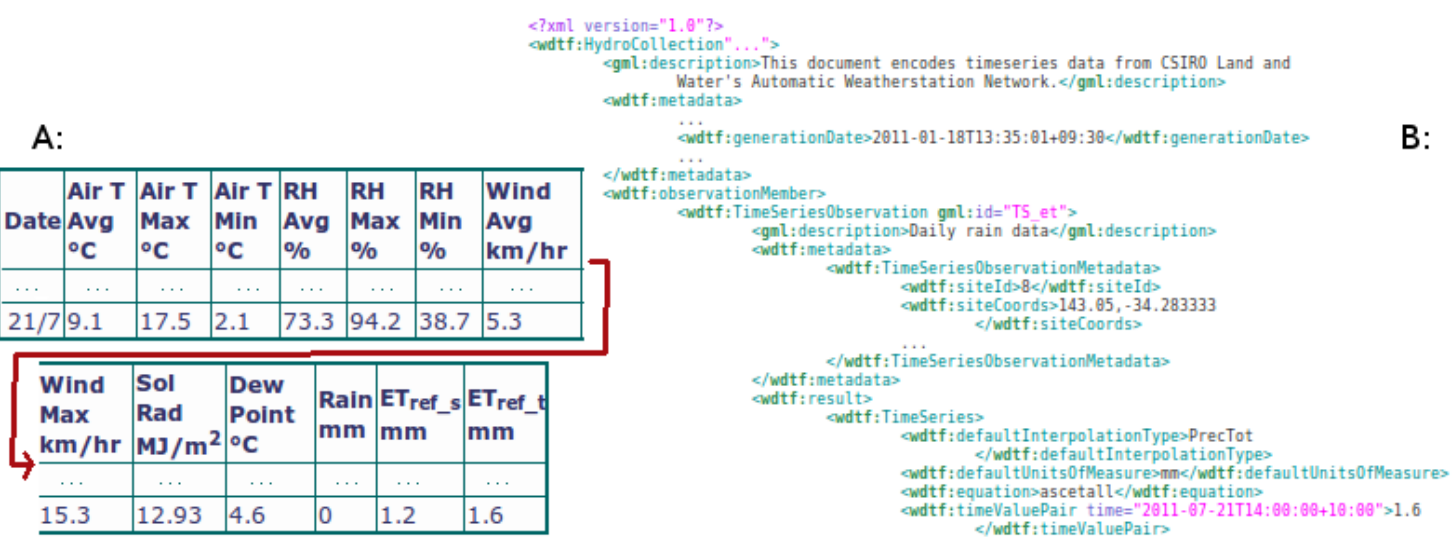

Figure 1. Data for the Griffith AWS for 21/01/11 in online visual HTML (A:) and WDTF (B:) formats

Network 2, South Australian MDB NRM Board: this network consists of around 50 AWSs in South Australia. Data was formerly available via a web page at http://www.aws-samdbnrm.sa.gov.au and also via dial-up download using proprietary software written by the weathestation hardware vendor. ${ }^{7}$. After standardisation, WDTF-like data was made available via FTP download from the network's central server. The files were updated every hour as new readings were received. Daily WDTF and modified WDTF data for this network are given at http://www.aws-samdbnrm.sa.gov.au/wdtf.

Before standardisation work, differences in underlying data and data delivery of these two networks would prevent a model or DSS easily using their data and separate data parser routines must be written for each. Standardisation allows a single WDTF reader module, perhaps freely distributed, to do so.

\subsection{Weather data from a single AWS}

Data taken directly from a single AWS supplied by a well-known Australian AWS manufacturer, is supplied to a client computer (PC or server) via FTP, as per Figure 2. Data from the AWS is encoded in a binary format which is hard to read other than with the software supplied by the same company. If an additional code routine were implemented at the AWS's transmission unit, data could instead be encoded in plain text and in a WDTF-like format. Data from this AWS could then be delivered either to a storage location and then read or directly to a model or DSS that could accept incoming FTP connections. This is not an unreasonable modification as the additional transmission payload caused by the binary to text/WDTF change is negligible ${ }^{8}$ when using $3 \mathrm{G}+$ mobile networks for data transmission and model and DSS makers can be expected to handle the widely implemented FTP protocol. A live example of mocked-up data for such a single AWS is given at http://www.aws-samdbnrm.sa.gov.au/wdtf/single including RESTful implementations of WDTF-like data delivery.

This change would effectively cease the AWS vendor's monopoly on data supply and interpretation/display, a situation which is currently popular with some vendors and not with others (see Section 5 for more on this). Such a split would possibly foster competition in the data display field with persons able to purchase AWS hardware from one vendor and display software (or a model or DSS) from another.

\section{Non-Weather-only Case Studies}

Case 1, A PC-based DSS integrating soil moisture and AWS data: currently, there are a few companies in Australia that supply both soil moisture monitoring and AWS equipment. However, in irrigation areas

\footnotetext{
${ }^{7}$ The software was Magpie and the vendor was Measurement Engineering Australia (MEA)

${ }^{8}$ The gain in required bandwidth between binary and marked-up, textual data is 5 times the original but for hourly transmissions over cellular networks, this payload cost is small compared with contract and connection fees.
} 


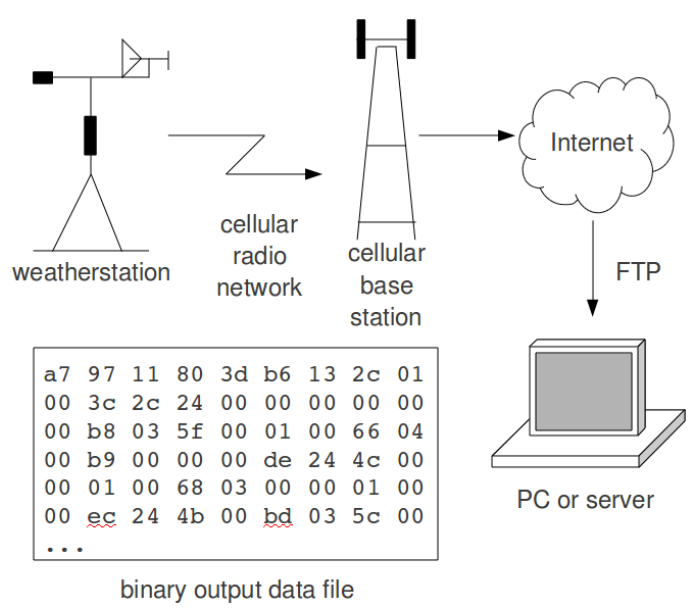

Figure 2. A single AWS to PC/server link and output binary data

such as South Australia's River Murray, government AWS cover is close to ubiquitous. An irrigator could thus buy soil moisture monitoring equipment and use it with the nearest government AWS if both used a standards-based format. Third party software developers also could build enhanced visualisation front ends to utilise existing soil moisture and weather data sensor installations.

Case 2, Heterogeneous sensor network: If the Queensland Government intends to use a large number of irrigators' existing water flow sensors for large area monitoring, it is certain that they will be represented by different brands. In this instance, no manufacturer's software will cover visualisation of all the sensors unless all brands are standards compliant. Indeed with standards compliance, as in Case 1, a third party application vendor may be able to easily build custom visualisation software.

\section{Industry Participation}

The, albeit very small, DSS industry in Australia is very integrated with respect to the information pathway from the sensor(s) to the user interface. For example, SENTEK ${ }^{9}$ manufactures probes and interface software for those probes that can be considered a DSS and currently one is not easily able to be used without the other. Indeed the author's own irrigation DSS, IrriSatSMS Hornbuckle et al. (2009), used an interface that was tied to a particular AWS network and additional AWSs or indeed other any sensors could not easily be added.

Several companies in the irrigation sensor sector have indicated a willingness to work towards a standard which, although they believe it might de-couple parts of the irrigation data delivery field which they currently hold market share across, they see as inevitable. If done correctly, standards compliance by them may open markets for their products both with large government projects in Australia who will work with organisations like the BoM and CSIRO and also overseas, especially when WaterML 2.0 is set.

\section{ACKNOWLEDGEMENTS}

The authors thanks the following companies and government entities who have informed thinking in this paper. Without their cooperation, the requirements for a standard relevant to irrigation could not easily be assessed.

- SENTEK - soil moisture monitoring probe manufacturer

- Water Data Services - hydrometric consultancy

- SA MDB NRM Board - South Australian Murray-Darling Basin NRM Board

The authors also thank Drs Evan Christen and John Hornbuckle at CSIRO Land \& Water in Griffith, NSW, for their support.

${ }^{9}$ See http://www.sentek.com.au/home/default.asp. 


\section{Conclusion}

This paper is intended, together with the live resource demonstrations listed in it and its presentation in Perth, as a reference point for irrigation researchers and industry regarding data source output standardisation in Australia. It is clear that these modifications to existing standards are needed but much can be done right now to start science and industry moving towards standards use. The hope is that this will lead to greater model and DSS use by irrigators which may in turn allow both water use efficiency increases and researchers to gather meaningful data more easily.

For direction, discussion needs to occur between irrigation industry equipment manufacturers, irrigation DSS and model designers, the BoM and other government agencies related to water use. CSIRO would provide a natural starting point for this discussion due to its dominance of DSS and model design, involvement in WDTF and sensor technologies.

\section{REFERENCES}

Allen, R. G., L. S. Pereira, D. Raes, and M. Smith (1998). Crop evapotranspiration - guidelines for computing crop water requirements. Irrigation and Drainage paper 56, FAO, FAO, Rome.

APSRU (2011, July). The agricultural production systems simulator (apsim). http://www.apsim.info.

Car, N. J., E. W.Christen, J. W. Hornbuckle, and G. A. Moore (2009). Irrigation modelling language for decision support. In R. B. Anderssen, R.S. and L. Newham (Eds.), 18th World IMACS Congress and MODSIM09 International Congress on Modelling and Simulation, pp. 484-490. Modelling and Simulation Society of Australia and New Zealand and International Association for Mathematics and Computers in Simulation.

C'wealth Gov't of Aust (2007, July). Commonwealth water act 2007. http://www.comlaw.gov.au/Details/C2007A00137.

Hornbuckle, J. W., N. J. Car, E. W. Christen, T. M. Stein, and B. Williamson (2009, April). Irrisatsms - irrigation water management by satellite and sms - a utilisation framework. Science Report 04/09, CSIRO Land \& Water, CSIRO Land and Water, Griffith, NSW, Australia.

Inman-Bamber, N. G., S. A. Attard, C. Baillie, D. Lawson, and L. Simpson (2005, May). A web-based system for planning use of limited irrigation water in sugarcane. In Proc. 2005 Conf. of the Aust. Soc. of Sugar Cane Technologists, Bundaberg, Queensland, Australia, pp. 170-181.

Shuttleworth, W. and J. Wallace (2009). Calculating the water requirements of irrigated crops in australia using the matt-shuttleworth approach. Transactions of the ASABE 52(6), 1895-1906.

Taylor, P. (2009). WDTF, WaterML2.0 \& water services. presentation online: http://www.kisters.de/service/downloadfile ?Open\&l=en\&id=F0A20F9EBC517A4DC12576730041292E.

Walker, G., P. Taylor, S. Cox, and P. Sheahan (2009). Water data transfer format (wdtf): Guiding principles, technical challenges and the future. In R. B. R.S. Anderssen and L. Newham (Eds.), 18th World IMACS Congress and MODSIM09. MSSANZ and International Assoc. for Mathematics and Computers in Simulation.

Walter, I., R. Allen, R. Elliott, M. Jensen, D. Itenfisu, B. Mecham, T. Howell, S. Snyder, P. Brown, S. Echings, T. Spofford, M. Hattendorf, R. Cuenca, J. Wright, and D. Martin (2000, November). Asce's standardized reference evapotranspiration equation. In Proceedings of Fourth National Irrigation Symposium ASAE, Phoenix, AZ, USA.

Zaslavsky, I., D. Valentine, and T. W. (Eds.) (2007). CUAHSI WaterML v0.3.0. http : //portal.opengeospatial.org/files/?artifact ${ }_{i} d=21743 S$. 